\title{
Search for resonant absorption of solar axions emitted in M1 transition in ${ }^{57} \mathrm{Fe}$ nuclei
}

\author{
A.V. Derbin ${ }^{\text {a }, ~ A . I . ~ E g o r o v, ~ I . A . ~ M i t r o p o l ' s k y, ~ V . N . ~ M u r a t o v a, ~ D . A . ~ S e m e n o v, ~ E . V . ~ U n z h a k o v ~}$ \\ St. Petersburg Nuclear Physics Institute, 188300 Gatchina, Russia
}

Received: 26 May 2009 / Revised: 17 June 2009 / Published online: 9 July 2009

(C) Springer-Verlag / Società Italiana di Fisica 2009

\begin{abstract}
A search for resonant absorption of $14.4 \mathrm{keV}$ solar axions by a ${ }^{57} \mathrm{Fe}$ target was performed. The $\mathrm{Si}(\mathrm{Li}) \mathrm{de}-$ tector placed inside the low-background setup was used to detect the $\gamma$-quanta appearing in the deexcitation of the 14.4 keV nuclear level: $A+{ }^{57} \mathrm{Fe} \rightarrow{ }^{57} \mathrm{Fe}^{*} \rightarrow{ }^{57} \mathrm{Fe}+\gamma$. The new upper limit for the hadronic axion mass has been obtained of $m_{A} \leq 159 \mathrm{eV}$ (95\% c.1.) $(S=0.5, z=0.56)$.
\end{abstract}

PACS $14.80 . \mathrm{Mz} \cdot 29.40 . \mathrm{Mc} \cdot 26.65 .+\mathrm{t}$

\section{Introduction}

A natural solution of the strong $\mathrm{CP}$ problem based on the global chiral symmetry U(1) was proposed by Peccei and Quinn [1]. Weinberg [2] and Wilczek [3] noted that spontaneous breaking of the PQ-symmetry at the energy $f_{A}$ leads to the existence of a new neutral spin-zero pseudoscalar particle-the axion. The axion mass $\left(m_{A}\right)$ and the strengths of an axion's coupling to an electron $\left(g_{A e}\right)$, a photon $\left(g_{A \gamma}\right)$ and nucleons $\left(g_{A N}\right)$ are proportional to the inverse of $f_{A}$. The original PQWW-model with $f_{A}$ fixed at the electroweak scale, $f_{A}=\left(\sqrt{2} G_{F}\right)^{-1 / 2}$, was excluded after intensive experimental searches which were performed using radioactive sources, reactors and accelerators (see [4] and refs therein).

New axion models decoupled the scale of the PQsymmetry breaking from the electroweak scale and the value of $f_{A}$ can be extended up to the Planck mass, $\approx 10^{19} \mathrm{GeV}$. Two classes of models for the "invisible" axion have been developed: the KSVZ or hadronic axion model $[5,6]$ and the DFSZ or GUT axion models [7, 8].

The axion mass in both models is given in terms of neutral pion properties:

$m_{A}=\frac{f_{\pi} m_{\pi}}{f_{A}}\left(\frac{z}{(1+z+w)(1+z)}\right)^{1 / 2}$,

a e-mail: derbin@pnpi.spb.ru where $f_{\pi} \cong 93 \mathrm{MeV}$ is the pion decay constant, $z=$ $m_{u} / m_{d} \approx 0.56$ and $w=m_{u} / m_{s} \approx 0.029$ are quark-mass ratios. For the given values of $z$ and $w(1)$ is numerically presented as $m_{A} \cong 6.0 \cdot 10^{6} / f_{A}$ where $m_{A}$ and $f_{A}$ are in $\mathrm{eV}$ and $\mathrm{GeV}$ units, respectively.

In contrast to the DFSZ axions, the KSVZ axions have no coupling to leptons and ordinary quarks at the tree level, which results in the strong suppression of the interaction of the KSVZ axion with electrons through radiatively induced coupling [9]. Moreover, in some models axion-photon coupling may differ from the original DFSZ or KSVZ $g_{A \gamma}$ couplings by a factor less than $10^{-2}[10]$.

The axion coupling constants are constrained by various experiments, astrophysical and cosmological arguments. The astrophysical limits based on the axion interaction strength with photons and electrons in stars suggest that $m_{A}<0.01 \mathrm{eV}$ if one assumes the standard $g_{A \gamma, A e}-m_{A}$ relations (see $[4,11]$ and refs therein).

The axion-nucleon coupling is constrained by the upper and lower limits based on the observed neutrino signal of SN1987A [12]. The supernova data leave open the so-called "hadronic axion window" of $m_{A} \sim 10 \mathrm{eV}$ if the $g_{A \gamma}$ coupling is sufficiently small. However, the cosmological limits on hot dark matter consisting of axions provide $m_{A}<1 \mathrm{eV}$ $[13,14]$.

If axions do exist, the Sun would be an intense source of these particles. Several main mechanisms of solar axion production are considered. Axions can be efficiently produced by Primakoff conversion of photons in the electric field of the plasma. The resulting axion flux has an average energy of about $4 \mathrm{keV}$ and can be detected by inverse Primakoff conversion in laboratory magnetic fields [15-18] or by the coherent conversion to photons in crystal detectors [19-22]. The experiments are sensitive to the $g_{A \gamma}$ coupling. The obtained upper limits for the axion-photon coupling constant are $g_{A \gamma} \leq 10^{-10} \div 10^{-8} \mathrm{GeV}^{-1}$, which still corresponds to the immense estimated axion flux at the level of $10^{11} \div 10^{13} \mathrm{~cm}^{-2} \mathrm{~s}^{-1} \mathrm{keV}^{-1}$. 
The other source of axions is the reactions of the solar cycle that produce solar energy [23]. Since axions are pseudoscalar particles they can be emitted in nuclear magnetic transitions. Attempts to detect $478 \mathrm{keV}$ monochromatic axions through the resonant absorption in a ${ }^{7} \mathrm{Li}$ nuclei target have been performed in [24-27].

Since the temperature in the center of the Sun is $\sim 1.3 \mathrm{keV}$, some nuclei having low-lying nuclear levels can be excited thermally [28]. Monochromatic axions can be emitted in the magnetic nuclear transitions from the first thermally excited level to the ground state. The aim of the present work is to search for the $14.4 \mathrm{keV}$ solar axions emitted by the M1 transition in ${ }^{57} \mathrm{Fe}$ nuclei. The axions on the Earth can be observed in the inverse reaction of the resonant absorption via the registration of $\gamma$-rays (or conversion electrons) produced by the discharge of the excited nuclear level [29]. The probability of emission and subsequent absorption of the axion in a magnetic transition is determined only by the axionnucleon coupling. The previous searches for $14.4 \mathrm{keV}$ axion were performed in [30-33].

\section{Emission and absorption of axions in nuclear transitions of magnetic type}

As has been found in [28], the most intense solar axion flux is connected with the M1 transition of a ${ }^{57} \mathrm{Fe}$ nucleus. The energy of the first excited nuclear level $3 / 2^{-}$is equal to $14.413 \mathrm{keV}$, and the admixture of the E2 transition is $\delta^{2}=0.22 \%$. The total axion flux $\Phi_{A}$ depends on the level energy $E_{\gamma}=14.413 \mathrm{keV}$, temperature, $T$, nuclear level lifetime, $\tau_{\gamma}=1.34 \mu$ s, the abundance of the ${ }^{57} \mathrm{Fe}$ isotope on the Sun, $N$, and the branching ratio of axions to photons emission, $\omega_{A} / \omega_{\gamma}[28,29]$ :

$\Phi_{A} \sim \frac{N}{\tau_{\gamma}} \frac{2 \exp \left(-E_{\gamma} / k T\right)}{\left(1+2 \exp \left(-E_{\gamma} / k T\right)\right)} \frac{\omega_{A}}{\omega_{\gamma}}$.

Owing to the Doppler broadening, the axion spectrum is a sum of Gaussian curves, $\Phi_{A}\left(E_{A}\right)$, with the dispersion $\sigma(T)=E_{\gamma}(k T / M)^{1 / 2}$, where $T$ is the temperature at the point where the axion is emitted and $M$ is the mass of the ${ }^{57} \mathrm{Fe}$ nucleus. The axion flux was calculated for the temperature dependence on the radius given by BS05(OP) Standard Solar Model [34] based on the corona high-Z abundances [35]. At the Earth, the differential axion flux at the maximum of the quasi-Gaussian distribution can be presented as

$\Phi_{A}\left(E_{M 1}\right)=4.15 \times 10^{25}\left(\frac{\omega_{A}}{\omega_{\gamma}}\right) \mathrm{cm}^{-2} \mathrm{~s}^{-1} \mathrm{keV}^{-1}$.

The obtained width of the axion line is equal to $\sigma_{S}=2.2 \mathrm{eV}$. This value significantly exceeds the energy of the recoilnucleus $(1.8 \mu \mathrm{eV})$, as well as the Doppler broadening of the line at temperature $T=300 \mathrm{~K}$ of the target nuclei $(10 \mathrm{meV})$, and the self-width of the level, $\Gamma=4.65 \cdot 10^{-9} \mathrm{eV}$ [36]. Thus, the percentage of axions satisfying the resonantabsorption condition amounts to the value $\sim \Gamma / \sigma_{S}$.

Within the framework of the long-wavelength approximation, the axion emission probability $\left(\omega_{A} / \omega_{\gamma}\right)$ is given by the expression $[28,37,38]$

$\frac{\omega_{A}}{\omega_{\gamma}}=\frac{1}{2 \pi \alpha} \frac{1}{1+\delta^{2}}\left[\frac{g_{A N}^{0} \beta+g_{A N}^{3}}{\left(\mu_{0}-0.5\right) \beta+\mu_{3}-\eta}\right]^{2}\left(\frac{p_{A}}{p_{\gamma}}\right)^{3}$,

where $p_{\gamma}$ and $p_{A}$ are the photon and axion momenta respectively; $\alpha \approx 1 / 137, \mu_{0} \approx 0.88, \mu_{3} \approx 4.71$ are the isoscalar and isovector nuclear magnetic moments, $\beta$ and $\eta$ are the parameters depending on the nuclear matrix elements. The values $\beta=-1.19$ and $\eta=0.8$ for the M1 transition in the ${ }^{57} \mathrm{Fe}$ nucleus were calculated in [28].

The interaction of the axion with nucleons is determined by the coupling constant $g_{A N}$, which consists of isoscalar $g_{A N}^{0}$ and isovector $g_{A N}^{3}$ parts. In the hadronic axion models, the $g_{A N}^{0}$ and $g_{A N}^{3}$ constants can be represented in the form $[9,10]$

$g_{A N}^{0}=-\frac{m_{N}}{6 f_{A}}\left[2 S+(3 F-D) \frac{1+z-2 w}{1+z+w}\right]$,

$g_{A N}^{3}=-\frac{m_{N}}{2 f_{A}}\left[(D+F) \frac{1-z}{1+z+w}\right]$.

Here, $m_{N}=939 \mathrm{MeV}$ is the nucleon mass, the constants $D$ and $F$ are expressed in terms of the isovector $\left(F_{A 3}\right)$ and isoscalar $\left(F_{A 0}\right)$ pion-nucleon coupling constants. The exact values of $D$ and $F$ parameters determined from the semileptonic hyperon decays are equal to $D=0.808 \pm 0.006$ and $F=0.462 \pm 0.011$ [39].

The parameter $S$ characterizing the flavor-singlet coupling still remains a poorly constrained one. Its value varies from $S=0.68$ in the naive quark model down to $S=-0.09$, which is given on the basis of the EMC collaboration measurements [40]. The more stringent boundaries $(0.37 \leq S \leq$ $0.53)$ and $(0.15 \leq S \leq 0.5)$ were found in [41] and [42], accordingly. As a result the value of the sum $\left(g_{A N}^{0} \beta+g_{A N}^{3}\right)$ in (4) may significantly decrease and, due to the negativity of the parameter $\beta$, actually vanishes. Taking into account that the usually accepted value of the $u$ - and $d$-quark-mass ratio $z=0.56$ can vary in the $0.35 \div 0.6$ range [4], the exact interpretation of the experimental results is significantly restricted. We use $S=0.5$ and $z=0.56$ as reference when calculating the axion flux for the KSVZ axion model.

According to (1), the constants $g_{A N}^{0}$ and $g_{A N}^{3}$ can be expressed in terms of the axion mass $(S=0.5)$ as

$g_{A N}^{0}=-4.03 \cdot 10^{-8}\left(m_{A} / 1 \mathrm{eV}\right)$,

$g_{A N}^{3}=-2.75 \cdot 10^{-8}\left(m_{A} / 1 \mathrm{eV}\right)$. 
The values of $g_{A N}^{0}$ and $g_{A N}^{3}$ for the DFSZ axion depend on the additional unknown parameter $\cos ^{2} \beta$, which is defined by the ratio of the Higgs vacuum expectation values, but they have the same order of magnitude $[9,10]$.

Because the branching ratio $\left(\omega_{A} / \omega_{\gamma}\right)$ is model dependent, we can consider the parameter $\left(g_{A N}^{0} \beta+g_{A N}^{3}\right)^{2}$ in the expression (4) as a free unknown parameter characterizing the axion-nucleon coupling.

The cross section of the resonant absorption of the axions is given by an expression similar to the one for the $\gamma$-ray absorption and corrected by the $\omega_{A} / \omega_{\gamma}$ ratio [43]:

$\sigma\left(E_{A}\right)=2 \sqrt{\pi} \sigma_{0 \gamma} \exp \left[-\frac{4\left(E_{A}-E_{M}\right)^{2}}{\Gamma^{2}}\right]\left(\frac{\omega_{A}}{\omega_{\gamma}}\right)$,

where $\sigma_{0 \gamma}$ is the maximum cross section of the $\gamma$-ray resonant absorption and $\Gamma=1 / \tau$. The experimentally obtained value of $\sigma_{0 \gamma}$ for the ${ }^{57} \mathrm{Fe}$ nucleus is equal to $2.56 \times$ $10^{-18} \mathrm{~cm}^{2}$ [44].

In order to obtain the total cross-section value, we should integrate the expression (9) over the axion spectrum given by (3). The integration of the narrow Gaussian distribution (9) over the wide axion spectrum yields a value close to $\Phi_{A}\left(E_{M 1}\right)$. Using the dependence of $\Phi_{A}$ (3) and $\sigma\left(E_{A}\right)$ (9) on the $\left(\omega_{A} / \omega_{\gamma}\right)$ ratio, and, therefore, on the axion mass $m_{A}$ $(4,7,8)$, one can numerically present the estimated rate of resonant absorption of axions by the ${ }^{57} \mathrm{Fe}$ nucleus $(S=0.5$, $z=0.56)$ :

$$
\begin{aligned}
R & =1.56 \cdot 10^{-3}\left(\omega_{A} / \omega_{\gamma}\right)^{2} \\
& =5.16 \cdot 10^{-3}\left(g_{A N}^{0} \beta+g_{A N}^{3}\right)^{4}\left(p_{A} / p_{\gamma}\right)^{6} \\
& =9.29 \cdot 10^{-34}\left(m_{A}\right)^{4}\left(p_{A} / p_{\gamma}\right)^{6} .
\end{aligned}
$$

The amount of detected $\gamma$-rays following the axion absorption is determined by the target mass, the time of measurement and the detector efficiency, while the observation probability for the $14.4 \mathrm{keV}$ peak depends on the background level of the experimental setup.

\section{Experimental setup}

The planar $\mathrm{Si}(\mathrm{Li})$ detector with the diameter of the sensitive region $17 \mathrm{~mm}$ and $2.5 \mathrm{~mm}$ thick was used for the detection of $14.4 \mathrm{keV} \gamma$-rays. The detector was mounted inside the vacuum cryostat with $2 \mu \mathrm{m}$ beryllium window. The $290 \mathrm{mg}$ iron target, enriched to $91 \%$ of ${ }^{57} \mathrm{Fe}$ isotope, was placed directly on the beryllium window; the distance between the detector's surface and the Fe target was $\approx 3 \mathrm{~mm}$. The surface density of the target was $92 \mathrm{mg} / \mathrm{cm}^{2}$, while the attenuation of $14.4 \mathrm{keV} \gamma$-rays in iron corresponds to $16 \mathrm{mg} / \mathrm{cm}^{2}$.

The detector was surrounded by $12.5 \mathrm{~cm}$ copper and $2.5 \mathrm{~cm}$ lead shields to eliminate the external $\gamma$-radioactivity.
The background level at $14.4 \mathrm{keV}$ was decreased by 110 times in comparison with the unshielded detector. The detector was located above the ground surface, so in order to minimize the influence of cosmic radiation and fast neutrons, we used the active shielding assembled of five $50 \times 50 \times 12 \mathrm{~cm}$ organic crystal scintillators. The neutron shielding and active muon veto cover the top and sides of the passive shield, except for the side where the detector Dewar is located. The anticoincidence veto signal was obtained from the logical OR of all the photomultiplier tube discriminator outputs. The $\mathrm{Si}(\mathrm{Li})$ detector operated in an anticoincidence mode with plastic scintillators and the background events around $14.4 \mathrm{keV}$ were reduced by a factor of 2.5 . The rate of $50 \mu \mathrm{s}$ veto signals was 600 counts/s, which leads to $\approx 3 \%$ dead time. The spectrum of the $\mathrm{Si}(\mathrm{Li})$ signals obtained in the coincidence with veto signals allows for checking the probability of the excitation $14.4 \mathrm{keV}$ level by the nuclear active component and cosmic ray muons.

The spectrometric channel of the $\mathrm{Si}(\mathrm{Li})$ detector was organized in the following way. The first-stage field-effect transistor was mounted on a teflon block, a few $\mathrm{mm}$ from the center contact of the silicon crystal, while the preamplifier was placed beyond the passive shielding. The data acquisition system was based on standard Camac electronics. A signal produced by the preamplifier was transmitted to two separate amplifiers with different gain ratios, thus providing the possibility to collect spectra from both lower $(0-60 \mathrm{keV})$ and higher energy $(0-500 \mathrm{keV})$ regions. The amplifier outputs were converted using analog-to-digital converters, controlled by a PC through parallel interfaces. Taking into account the active shielding coincidence spectra for each amplifier, there were four 4096-channel spectra being recorded.

The energy scale was defined using standard calibration sources of ${ }^{55} \mathrm{Fe},{ }^{57} \mathrm{Co}$ and ${ }^{241} \mathrm{Am}$. The energy resolution of the detector (FWHM) determined by the $14.4 \mathrm{keV} \gamma$-line of ${ }^{57} \mathrm{Co}$ source turned out to be $280 \mathrm{eV}$.

The detection efficiency of the $\mathrm{Si}(\mathrm{Li})$ detector was measured with $14.4 \mathrm{keV} \gamma$-rays from a standardized ${ }^{57} \mathrm{Co}$ source. The self-absorption of $14.4 \mathrm{keV} \gamma$-rays by the iron target was found via a detailed M-C simulation. The overall detection efficiency for $14.4 \mathrm{keV} \gamma$ is estimated to be $(2.30 \pm 0.1) \%$.

\section{Results}

The measurements were carried out during 84.5 days of live time by $\sim 2$-hour runs. The obtained energy spectra are given in Fig. 1. Since the ${ }^{57} \mathrm{Fe}$ target consisted of a small amount of ${ }^{55} \mathrm{Fe}$, the most intense peaks are connected with the characteristic X-rays of ${ }^{55} \mathrm{Mn}\left(\mathrm{K}_{\alpha}=5.9 \mathrm{keV}, \mathrm{K}_{\beta}=6.49 \mathrm{keV}\right)$. One can clearly identify several peaks related to the ${ }^{241} \mathrm{Am}$ radioactivity. The low-energy region contains the L-series 


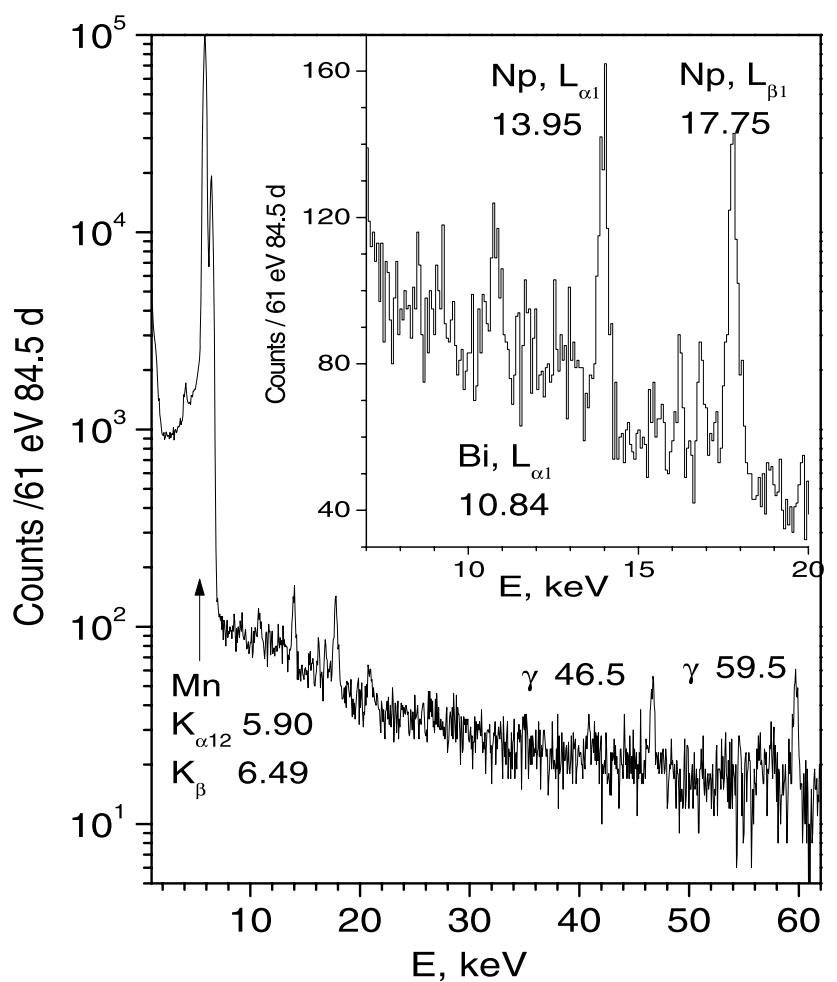

Fig. 1 Energy spectrum of the $\mathrm{Si}(\mathrm{Li})$ detector measured in the region $1-60 \mathrm{keV}$ (main) and 7-20 keV (inset)

of characteristic X-rays caused by ${ }^{241} \mathrm{Am} \rightarrow{ }^{234} \mathrm{~Np}^{*}+\gamma$ decay. The $13.9 \mathrm{keV}$ peak consists of two lines with the energies $13.946 \mathrm{keV}\left(13 \%, \mathrm{~L}_{\alpha 1}\right)$ and $13.761\left(1.4 \%, \mathrm{~L}_{\alpha 2}\right)$; the $17.8 \mathrm{keV}$ peak is the more complex one formed by $\mathrm{L}_{\beta 1-5}$ lines. The peaks with the energies of $10.84 \mathrm{keV}$ and $13.2 \mathrm{keV}\left(\mathrm{L}_{\alpha 1}\right.$ and $\mathrm{L}_{\beta 1-5}$ of $\mathrm{Bi}$, respectively) are present due to ${ }^{210} \mathrm{~Pb} \rightarrow{ }^{210} \mathrm{Bi} \beta$-decay in the ${ }^{238} \mathrm{U}$ series. The detection of the low-energy X-rays of ${ }^{234} \mathrm{~Np}$ and ${ }^{210} \mathrm{Bi}$ means that the sources of radioactivity are placed close to the sensitive volume of the $\mathrm{Si}(\mathrm{Li})$ detector. A small internal contamination of ${ }^{241}$ Am may be from previous usage, while the ${ }^{238} \mathrm{U}$ natural radioactivity can be contained in the holder of the detector or in the cryostat cup. Intense ${ }^{241} \mathrm{Am}(59.5 \mathrm{keV})$ and ${ }^{210} \mathrm{Bi}$ $(46.5 \mathrm{keV}) \gamma$-rays are observed at the higher energy region. All these peaks were used to determine the final energy scale and energy resolution $\sigma(\mathrm{E})$ of the detector.

Figure 2 shows the detailed energy spectrum within the 12.2-16.6 keV interval, where the axion peak was expected. Apparently, there is no pronounced peak at the $14.4 \mathrm{keV}$. In order to determine the upper limit for the number of events inside the expected peak we used the maximum likelihood approach. The likelihood function was determined as a sum of four Gaussians and the polynomial background. Three Gaussians represent the known characteristic X-rays, one Gaussian stands for the expected $14.4 \mathrm{keV}$ axion peak and a

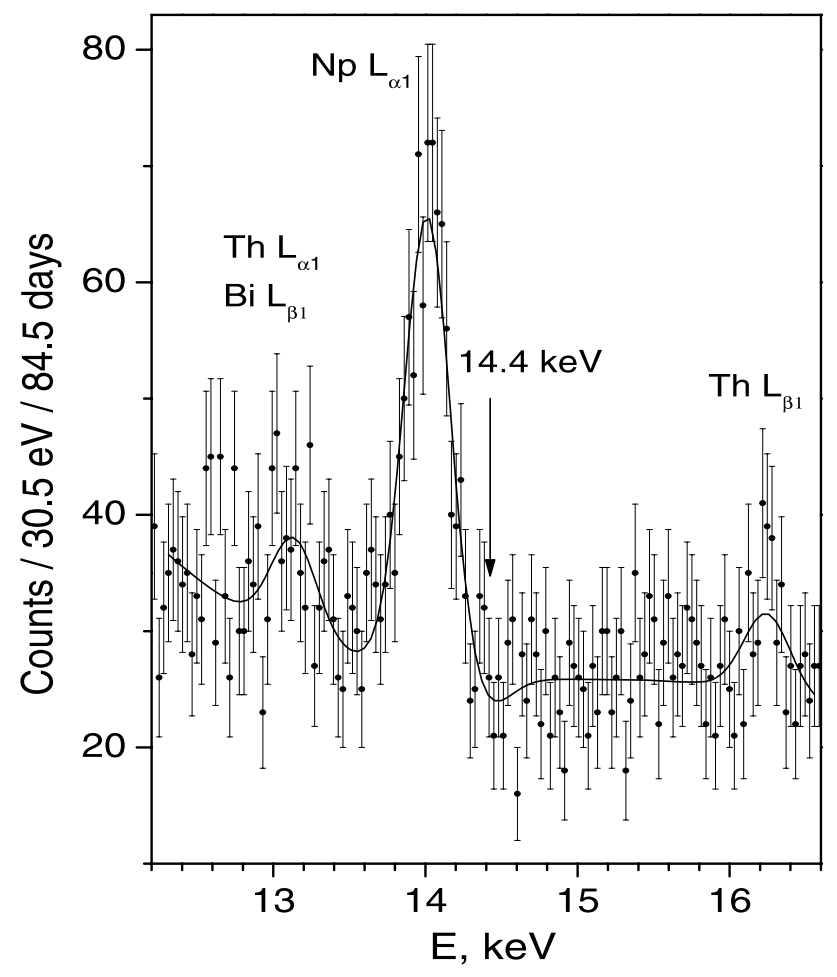

Fig. 2 Fitting results of the energy spectrum inside the $12.4-16.6 \mathrm{keV}$ region. The location of the expected axion peak is denoted by an arrow

third order polynomial is used for the smooth background:

$$
\begin{aligned}
N(E)= & a+b \cdot E+c \cdot E^{2}+d \cdot E^{3} \\
& +\frac{1}{\sqrt{2 \pi} \sigma} \sum_{i=1}^{4} S_{i} \exp \left[-\frac{\left(E_{i}-E\right)^{2}}{2 \sigma^{2}}\right] .
\end{aligned}
$$

Peak positions and energy resolution $(\sigma)$ were fixed, while the peak areas and background polynomial coefficients were independent free parameters. The total number of degrees of freedom at the 12.4-16.6 keV region amounted to 250 .

The fitting result is given in Fig. 2. The minimum $\chi^{2}$ value corresponds to the nonphysical value of the $14.4 \mathrm{keV}$ peak area of $S_{A}=-25$ events. The upper limit for the amount of events inside the peak was found via the conventional approach: the dependence of $\chi^{2}$ on the peak area $S_{A}$ was calculated for various values of $S_{A}$, while the rest parameters were left unrestrained. Then the appearance probability of the given $\chi^{2}\left(S_{A}\right)$ value was found and the obtained function $P\left(\chi^{2}\left(S_{A}\right)\right)$ was normalized to unity for the $S_{A} \geq 0$ region. Thus, the upper limit appeared to be equal to $S_{\text {lim }}=29$ events at $95 \%$ c.l. For the rate of axion absorption $R$ given by (10) the expected number of registered $14.4 \mathrm{keV}$ $\gamma$-quanta is

$S_{A}=\varepsilon \cdot \eta \cdot N \cdot T \cdot R \leq S_{\mathrm{lim}}$. 


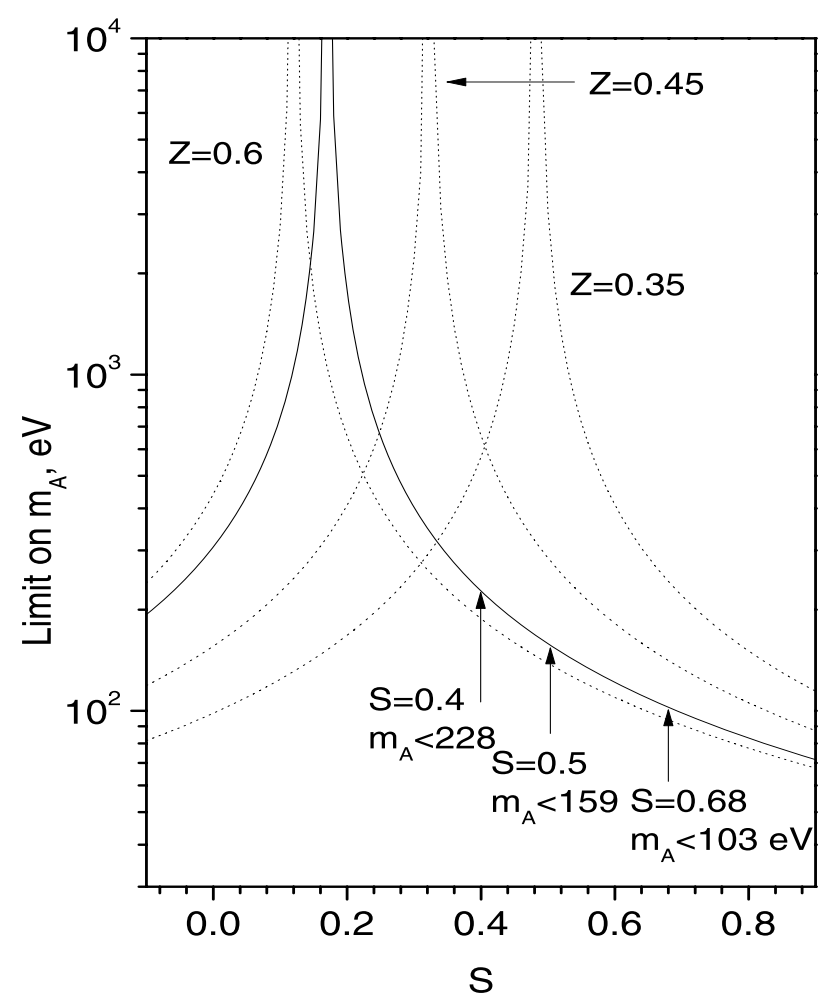

Fig. 3 The limit on the hadronic axion mass versus $S$ for various values of $z$ (95\% c.l.). The solid line corresponds to $z=0.56$

Here, the number of ${ }^{57} \mathrm{Fe}$ nuclei is $N=2.78 \cdot 10^{21}$, the measurement time $T=7.30 \cdot 10^{6} \mathrm{~s}$, the $\gamma$-ray registration efficiency $\varepsilon=2.3 \cdot 10^{-2}$ and the internal conversion ratio $\eta=0.105$.

The relation (14) limits possible values of the axionnucleon couplings constants and axion mass. In accordance with (10) and on condition that $\left(p_{A} / p_{\gamma}\right)^{6} \cong 1$ provided for $m_{A}<2 \mathrm{keV}$ one can obtain

$$
\begin{aligned}
& \left|-1.19 \cdot g_{A N}^{0}+g_{A N}^{3}\right| \leq 3.27 \cdot 10^{-6}, \quad \text { and } \\
& m_{A} \leq 159 \mathrm{eV} \quad \text { at } 95 \% \text { c.l. }
\end{aligned}
$$

The limit (16) is the strongest up-to-date result obtained with $14.4 \mathrm{keV}$ solar axions. The previous limit $\left(m_{A} \leq\right.$ $216 \mathrm{eV}$ [32]) is improved in $\approx 1.4$ time. Because the expected intensity of 14.4 peak depends on $m_{A}^{4}$, which in fact corresponds to an increase of the sensitivity of the experiment of more than $\approx 3$ times. The obtained limit on hadronic axion mass depends on the exact values of the parameters $S$ and $z$ (Fig. 3). The uncertainty of the flavor-singlet axialvector matrix element $S$ changes the obtained constraints significantly: $m_{A} \leq 228 \mathrm{eV}(S=0.4)$ and $m_{A} \leq 103 \mathrm{eV}$ ( $S=0.68$ ). Moreover, if the value of $S$ is close to 0.17 , the limit on the hadronic axion mass cannot be derived from the present experiment.

The value of $u$ - and $d$-quark-mass ratio $z=0.56$ is generally accepted for axion papers, but it could vary in the range $0.35 \div 0.6$ [4]. For the fixed $S=0.5$, the changes of $z$ from 0.6 to 0.45 lead to the limits $m_{A} \leq(139 \div 285) \mathrm{eV}$ correspondingly. If $z \cong 0.34$, the hadronic axion mass can not be restricted by the experiment. The reason is that the ratio $\left(\omega_{A} / \omega_{\gamma}\right)$ vanishes for some values of $z$ and $S$. One can obtain from (4) and (5) that if $S$ and $z$ obey the relation $S=1-1.5 \cdot(z \pm 0.01)$, the limit on the axion mass can not be obtained. Nevertheless, the limit on the axion-nucleon coupling given by (15) is still valid.

As mentioned above, the main disadvantage of the approach with axions emitted in the $14.4 \mathrm{keV} \mathrm{M} 1$ transition of ${ }^{57} \mathrm{Fe}$ is that the nuclear-structure-dependent parameter $\beta$ has a negative value which, together with the poorly constrained flavor-singlet axial-vector matrix element $S$, leads to large uncertainty of the $\left(\omega_{A} / \omega_{\gamma}\right)$ ratio.

\section{Conclusion}

A search for resonant absorption of the solar axion by ${ }^{57} \mathrm{Fe}$ nuclei was performed using the planar $\mathrm{Si}(\mathrm{Li})$ detector installed inside the low-background setup. The intensity of the $14.4 \mathrm{keV}$ peak measured for 84.5 days turned out to be $\leq 0.28$ events/day. The obtained model-independent upper limit on axion-nucleon couplings is $\left|g_{A N}^{0} \beta+g_{A N}^{3}\right| \leq$ $3.27 \cdot 10^{-6}$, which allowed us to set the new upper limit on the hadronic axion mass of $m_{A} \leq 159 \mathrm{eV}$ (95\% c.l.) with the generally accepted values $S=0.5$ and $z=0.56$. The obtained limit on the axion mass strongly depends on the exact values of the parameters $S$ and $z$.

\section{References}

1. R.D. Peccei, H.R. Quinn, Phys. Rev. Lett. 38, 1440 (1977)

2. S. Weinberg, Phys. Rev. Lett. 40, 223 (1978)

3. F. Wilczek, Phys. Rev. Lett. 40, 279 (1978)

4. C. Amsler et al. (Particle Data Group), Phys. Lett. B 667, 1 (2008)

5. J.E. Kim, Phys. Rev. Lett. 43, 103 (1979)

6. M.A. Shifman, A.I. Vainstein, V.I. Zakharov, Nucl. Phys. B 166, $493(1980)$

7. A.R. Zhitnitskii, Yad. Fiz. 31, 497 (1980) [Sov. J. Nucl. Phys. 31, 260 (1980)]

8. M. Dine, F. Fischler, M. Srednicki, Phys. Lett. B 104, 199 (1981)

9. M. Srednicki, Nucl. Phys. B 260, 689 (1985)

10. D.B. Kaplan, Nucl. Phys. B 260, 215 (1985)

11. G.G. Raffelt, arXiv:hep-ph/0611350

12. G.G. Raffelt, Ann. Rev. Nucl. Phys. Sci. 49, 163 (1999)

13. S. Hannestad et al., arXiv:0706.9148

14. G. Raffelt et al., arXiv:0808.0814

15. Y. Inoue et al. (Tokyo helioscope), Phys. Lett. B 536, 18 (2002)

16. Y. Inoue et al. (Tokyo helioscope), Phys. Lett. B 668, 93 (2008)

17. K. Zioutas et al. (CAST Collaboration), Phys. Rev. Lett. 94, 121301 (2005). arXiv:0810.4482

18. E. Arik et al. (CAST Collaboration), J. Cosmol. Astropart. Phys. 0902.008 (2009). arXiv:0810.4482

19. F.T. Avignone et al. (Solax Collaboration), Nucl. Phys. (Proc. Supll.) 72, 176 (1999) 
20. R. Bernabei et al. (DAMA Collaboration), Phys. Lett. B 515, 6 (2001)

21. A. Morales et al. (Cosme Collaboration), Astropart. Phys. 16, 325 (2002)

22. Z. Ahmed et al. (CDMS Collaboration), arXiv:0902.4693 (2009)

23. G. Raffelt, L. Stodolsky, Phys. Lett. B 119, 323 (1982)

24. M. Krçmar et al., Phys. Rev. D 64, 115016 (2001)

25. A.V. Derbin et al., JETP Lett. 81, 365 (2005)

26. G. Bellini et al. (Borexino Collaboration), Eur. Phys. J. C 54, 61 (2008)

27. P. Belli et al., Nucl. Phys. A 806, 388 (2008)

28. W.C. Haxton, K.Y. Lee, Phys. Rev. Lett. 66, 2557 (1991)

29. S. Moriyama, Phys. Rev. Lett. 75, 3222 (1995)

30. M. Krçmar et al., Phys. Lett. B 442, 38 (1998)

31. A.V. Derbin et al., JETP Lett. 85, 12 (2007)

32. T. Namba, Phys. Lett. B 645, 398 (2007)
33. A.V. Derbin et al., Bull. Rus. Acad. Sci. Phys. 71, 832 (2007)

34. J.N. Bahcall, A.M. Serenelli, S. Basu, Astrophys. J. 621, L85 (2005)

35. N. Grevesse, A.J. Sauval, Space Sci. Rev. 85, 161 (1998)

36. C.M. Lederer, V.S. Shierley, Table of Isotopes (Wiley, New York, 1978)

37. T.W. Donnelly et al., Phys. Rev. D 18, 1607 (1978)

38. F.T. Avignone III et al., Phys. Rev. D 37, 618 (1988)

39. V. Mateu, A. Pich, J. High Energy Phys. 10, 41 (2005)

40. R. Mayle et al., Phys. Lett. B 219 (1989)

41. G. Altarelli et al., Phys. Lett. B 496, 337 (1997)

42. D. Adams et al., Phys. Rev. D 56, 5330 (1997)

43. A. De Rújula, K. Zioutas, Phys. Lett. B 217, 354 (1989)

44. D.P. Johnson, Phys. Rev. B 1, 3551 (1970). Mossbauer effect data center, e.g. http://orgs.unca.edu/medc/ 